\title{
APPROXIMATING TOPOLOGICAL METRICS BY RIEMANNIAN METRICS
}

\author{
STEVEN C. FERRY AND BORIS L. OKUN
}

(Communicated by James West)

\begin{abstract}
We study the relation between (topological) inner metrics and Riemannian metrics on smoothable manifolds. We show that inner metrics on smoothable manifolds can be approximated by Riemannian metrics. More generally, if $f: M \rightarrow X$ is a continuous surjection from a smooth manifold to a compact metric space with $f^{-1}(x)$ connected for every $x \in X$, then there is a metric $d$ on $X$ and a sequence of Riemannian metrics $\left\{\psi_{i}\right\}$ on $M$ so that $\left(M, \psi_{i}\right)$ converges to $(X, d)$ in Gromov-Hausdorff space. This is used to obtain a (fixed) contractibility function $\rho$ and a sequence of Riemannian manifolds with $\rho$ as contractibility function so that $\lim \left(M, \psi_{i}\right)$ is infinite dimensional. Using results of Dranishnikov and Ferry, this also gives examples of nonhomeomorphic manifolds $M$ and $N$ and a contractibility function $\rho$ so that for every $\varepsilon>0$ there are Riemannian metrics $\phi_{\varepsilon}$ and $\psi_{\varepsilon}$ on $M$ and $N$ so that $\left(M, \phi_{\varepsilon}\right)$ and $\left(N, \psi_{\varepsilon}\right)$ have contractibility function $\rho$ and $d_{G H}\left(\left(M, \phi_{\varepsilon}\right),\left(N, \psi_{\varepsilon}\right)\right)<\varepsilon$.
\end{abstract}

Definition 1. A map $f: M \rightarrow X$ from a topological manifold onto a compact metric space is said to be

(1) $U V^{0}$ if $f$ is surjective and $f^{-1}(m)$ is connected for each $m \in M$.

(2) Cell-like or $C E$ if for each $x \in X$ and neighborhood $U$ of $f^{-1}(x)$ there is a neighborhood $V \subset U$ of $f^{-1}(x)$ so that the inclusion $V \rightarrow U$ is null homotopic.

CE maps are, more-or-less, surjections with contractible point-inverses. See [L] for general information on $U V^{0}$ and CE maps. One checks easily from the definition that CE maps are $U V^{0}$.

Definition 2. A function $\rho:[0, R] \rightarrow[0, \infty)$ which is continuous at 0 with $\rho(0)=0$ and $\rho(t) \geq t$ for all $t$ is called a contractibility function.

(1) A metric space $X$ has contractibility function $\rho$ if for each $x \in X$ and $t \leq R$, the metric ball of radius $t$ centered at $x$ contracts to a point in the concentric ball of radius $\rho(t)$.

Received by the editors September 18, 1993.

1991 Mathematics Subject Classification. Primary 53C23, 57N60, 57R12.

Key words and phrases. Riemannian manifold, length space, cell-like map.

The first author was partially supported by NSF Grant DMS9305758. 
(2) If $\rho$ is a contractibility function, we will say that $X \in L G C(n, \rho)$ if for each map $\alpha: S^{k} \rightarrow X, k \leq n$, with $\operatorname{diam}\left(\alpha\left(S^{k}\right)\right)<R$ there is a map $\bar{\alpha}: D^{k+1} \rightarrow X$ extending $\alpha$ with $\operatorname{diam}\left(\bar{\alpha}\left(D^{k+1}\right)\right)<\rho\left(\operatorname{diam}\left(\alpha\left(S^{k}\right)\right)\right)$. We will say that $X \in L G C(n)$ if $X \in L G C(n, \rho)$ for some $\rho$.

In what follows, $\mathscr{C} \mathscr{M}$ will refer to the Gromov-Hausdorff space of isometry classes of compact metric spaces. The main theorem of [Mo] shows that if $M$ is a topological manifold and $f: M \rightarrow X$ is $\mathrm{CE}$, then there is a path $\omega:[0,1] \rightarrow$ $\mathscr{C} \mathscr{M}$ so that

(1) $\omega(t)$ is homeomorphic to $M$ for $0 \leq t<1$.

(2) $\omega(1)=X$.

(3) There is a contractibility function $\rho$ so that $\rho$ is a contractibility function for $\omega(t)$ for $0 \leq t<1$.

Following work of Dranishnikov and Edwards (see [DW] for references), Dydak and Walsh have constructed a CE map $f: S^{5} \rightarrow X$ where $X$ is infinite dimensional. Moore's theorem therefore shows that there is a sequence of topological metrics $\left(S_{i}, d_{i}\right)$ on $S^{5}$ with a common contractibility function $\rho$ so that $\lim _{i \rightarrow \infty} S_{i}$ is infinite dimensional. The goal of this note is to exhibit sequences of Riemannian metrics (including a sequence on $S^{5}$ ) with the same behavior.

Definition 3. Bing calls a metric space $(X, d)$ convex if for every $x, y \in X$ there is a $z \in X$ so that $d(x, z)=d(z, y)=\frac{1}{2} d(x, y)$. If $X$ is convex and $x, y \in X$ with $d=d(x, y)$, then there is an isometric embedding $i:[0, d] \rightarrow$ $X$ with $i(0)=x$ and $i(d)=y$. More recent literature refers to these compact convex metric spaces as compact inner metric spaces or path spaces.

According to Bing (see [B]) every compact, connected metrizable space in $L G C(0)$ has an inner metric. Continuous images of connected topological manifolds are $L G C(0)$, so all continuous images of manifolds possess inner metrics. By [D], every locally simply connected compactum is the $U V^{0}$ image of a manifold.

Here is our main result.

Theorem. Let $f: M \rightarrow X$ be a $U V^{0}$-map from a closed connected smooth manifold $M, \operatorname{dim} M>2$, onto a space $X$. If $d$ is a convex metric on $X$, then:

(i) There is a sequence $\left\{\psi_{i}\right\}$ of Riemannian metrics on $M$ so that the metric space $(X, d)$ is the Gromov-Hausdorff limit of the sequence $\left(M, \psi_{i}\right)$.

(ii) If the map $f$ is cell-like, then the metrics $\left\{\psi_{i}\right\}$ can be chosen to have a common contractibility function $\rho$.

Remark 4. $U V^{0}$-maps are not hard to come by. If $X$ is a connected polyhedron (or ANR), there is a $U V^{0}$-map from a connected manifold $M^{n}$ to $X, n \geq 3$, if and only if there is a map $f: M \rightarrow X$ which is surjective on $\pi_{1}$. See [Be, F] for these results and for classical references. Our theorem can therefore be combined with Bing's theorem to show that each compact ANR can be obtained as the Gromov-Hausdorff limit of a single closed 3-manifold (which depends on the choice of ANR) with varying Riemannian metric. The 1-skeleton of the nerve of a fine cover of $X$ maps to $X$ and gives a surjection on $\pi_{1}$. Thickening this 1-complex to a regular neighborhood in $\mathbb{R}^{4}$ and restricting to the boundary gives a map from a closed 3-manifold to $X$ which is surjective on $\pi_{1}$ and which is therefore homotopic to a $U V^{0}$-map. Alternatively, we could take one of the 


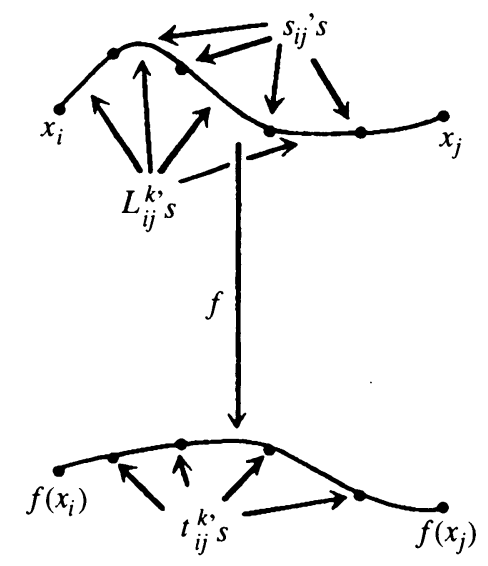

FIGURE 1

approximating 2-manifolds from [C], cross with $S^{1}$ to raise the dimension, and homotop that map to a $U V^{0}$-map.

The appearance of $U V^{0}$ maps in these constructions is not accidental. The last section of $[\mathrm{C}]$ can be combined with the results of $[\mathrm{Be}, \mathrm{F}]$ referred to above to show that a Riemannian manifold sufficiently close to an ANR in GromovHausdorff space admits a $U V^{0}$-map to that ANR.

Proof of Theorem. By [W], we may assume that the map $f$ is open. We denote the diameter of $(X, d)$ by $D$. Fix an $\varepsilon>0$ and let $h=\sqrt{2 D \varepsilon+\varepsilon^{2}}$. We will show that there exist a Riemannian metric $\psi$ on $M$ and $\varepsilon$-isometric $(2 h+5 \varepsilon)$ dense $^{1}$ sets in $(M, \psi)$ and $(X, d)$. This suffices to establish part (i) of our theorem.

Let $\phi^{\prime}$ be some Riemannian metric on $M$. We begin by rescaling the metric $\phi^{\prime}$ to a metric $\phi$ so that

$$
\phi(x, y)<d(f(x), f(y))-\varepsilon
$$

for all $x, y \in M$. Let $\delta>0$ be less than the injectivity radius of $\phi$ and small enough so that $d(f(x), f(y))<\varepsilon$ whenever $\phi(x, y)<\delta$. Choose $\left\{x_{i}\right\}$ to be an $\varepsilon$-dense set in $(M, \phi)$ so that for each $x \in M$ there is an $x_{i} \in M$ so that $\phi\left(x_{i}, x\right)<\varepsilon$ and $d\left(f(x), f\left(x_{i}\right)\right)<\varepsilon$. We also require $f\left(x_{i}\right) \neq f\left(x_{j}\right)$ for $i \neq j$. This uses the openness of $f$.

Let $l_{i j}$ be a minimal geodesic in $X$ connecting $f\left(x_{i}\right)$ and $f\left(x_{j}\right)$. Since the map $f$ is open and $U V^{0}$, we can subdivide each $l_{i j}$ using vertices $t_{i j}^{k}$ and find lifts $s_{i j}^{k}$ of the $t_{i j}^{k}$ 's so that $\phi\left(s_{i j}^{k}, s_{i j}^{k+1}\right)<\delta$. We choose geodesics $L_{i j}^{k}$ connecting adjacent vertices $s_{i j}^{k}$ and $s_{i j}^{k+1}$ (see Figure 1). Since $\operatorname{dim} M \geq 3$, we can arrange that these geodesics have disjoint interiors. We write $L_{i j}=\bigcup_{k} L_{i j}^{k}$.

Consider a very thin tubular neighborhood around $\bigcup L_{i j}$. By changing $\phi$ inside this neighborhood we get an even smaller tubular neighborhood such that the metric over each $L_{i j}^{k}$ is a product $L_{i j}^{k} \times B_{r}$ of $L_{i j}^{k}$ with a ball of some small radius $r$. In particular, $r$ should be much smaller than the injectivity radius

\footnotetext{
${ }^{1} \mathrm{~A}$ subset $C \subset Z$ is $\varepsilon$-dense if for each $z \in Z$ there is some $c \in C$ with $d(z, c)<\varepsilon$.
} 

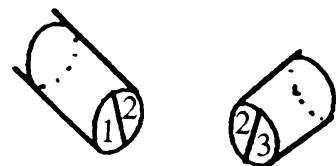

II

FIGURE 2
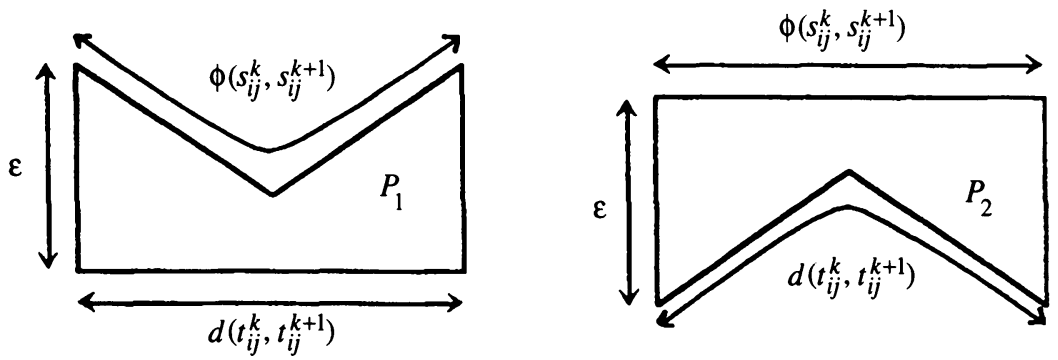

FIGURE 3

of $M$. At the ends of the $L_{i j}$ 's, we paste the cylinders together by dividing the disks at the ends in half and pasting the halves together in an alternating pattern (see Figure 2). Because we are not folding our disks along the plane dividing the halves, the resulting piecewise Riemannian metric is singular on planes perpendicular to a codimension-two submanifold. We can do this so that $\left\{x_{i}\right\}$ is still an $\varepsilon$-dense set and so that condition $(*)$ still holds.

Next, we modify the metric inside these cylinders. Inside of the cylinder $L_{i j}^{k} \times B_{r / 4}$ we change the metric to make it isometric to $\left[0, d\left(t_{i j}^{k}, t_{i j}^{k+1}\right)\right] \times B_{r}$. On the cylindrical annulus $L_{i j}^{k} \times\left(B_{r / 2}-B_{r / 4}\right)$ we put the metric of $T \times S_{r}$ where $T$ is either the nonconvex pentagon $P_{1}$ shown in Figure 3 or a rectangle $\left[0, d\left(t_{i j}^{k}, t_{i j}^{k+1}\right)\right] \times[0, \varepsilon]$ according to whether $\phi\left(s_{i j}^{k}, s_{i j}^{k+1}\right)$ is greater or less than $d\left(t_{i j}^{k}, t_{i j}^{k+1}\right)$.

On the cylinderical annulus $L_{i j}^{k} \times\left(B_{r}-B_{3 / 4 r}\right)$ we put the metric of $T^{\prime} \times S_{r}$ where $T^{\prime}$ is again either a nonconvex pentagon or a rectangle $\left[0, \phi\left(s_{i j}^{k}, s_{i j}^{k+1}\right)\right] \times$ $[0, \varepsilon]$ according to whether $\phi\left(s_{i j}^{k}, s_{i j}^{k+1}\right)$ is less or greater than $d\left(t_{i j}^{k}, t_{i j}^{k+1}\right)$. On the cylindrical annulii $L_{i j}^{k} \times\left(B_{3 / 4 r}-B_{r / 2}\right)$, which together give a collar on the boundary of the tubular neighborhood, we put the metric of

$$
\left[0, \max \left(\phi\left(s_{i j}^{k}, s_{i j}^{k+1}\right), d\left(t_{i j}^{k}, t_{i j}^{k+1}\right)\right)\right] \times[0, h] \times S_{r} .
$$

The effect of this is to build a "vertical" barrier of height $h$ between the tubular neighborhood and the rest of the manifold (see Figure 4).

Smoothing this piecewise Riemannian metric gives the metric $\psi$ on $M$ that we are looking for. First, let us show that $\psi$ satisfies inequality

$$
\psi(x, y)>d(f(x), f(y))-\varepsilon .
$$




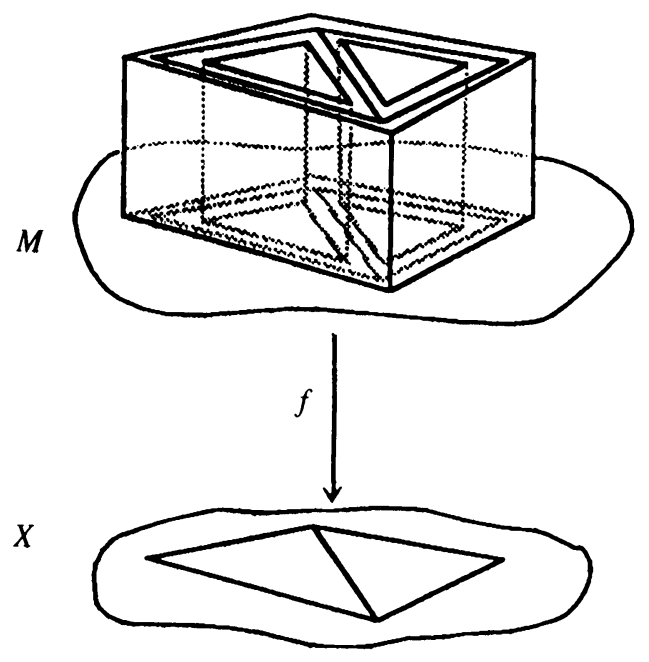

FIGURE 4

Let $s$ be any piecewise smooth curve in $M$ connecting two points $a$ and $b$. If $s$ lies in the complement of the $r / 2$-neighborhood (in the metric $\phi$ ) of $\bigcup L_{i j}$, then, since $\psi$ is bigger than $\phi$ there, the inequality for the length of $s$ holds because of $(*)$. On the other hand, if $s$ lies inside of our $\frac{3}{4} r$-neighborhood, then we have $l_{\psi}(s ; a, b) \geq d\left(t_{i j}^{k}, t_{i j}^{k+1}\right) \geq d(f(a), f(b))-\varepsilon$. To prove $(* *)$ in general, we break the curve into pieces inside and outside of the neighborhood and note that if the length of $s$ is less than $D$, then by the Pythagorean theorem each crossing of the region between $r / 2$ and $3 / 4 r$ adds an extra $\varepsilon$ in the new metric.

It follows from $(* *)$ that $\left|\psi\left(x_{i}, x_{j}\right)-d\left(f\left(x_{i}\right), f\left(x_{j}\right)\right)\right|<\varepsilon$ for all $i, j$, so the finite metric spaces $\left\{x_{i}\right\}$ and $\left\{f\left(x_{i}\right)\right\}$ are $\varepsilon$-close in the Gromov-Hausdorff metric. Since $\left\{f\left(x_{i}\right)\right\}$ was chosen to be $\varepsilon$-dense in $(X, d)$, this shows that $\left\{x_{i}\right\}$ is $2 \varepsilon$-close to $(X, d)$ in Gromov-Hausdorff space. Let us now show that $\left\{x_{i}\right\}$ is $(2 h+5 \varepsilon)$-dense in $(M, \psi)$. This will show that the Gromov-Hausdorff distance from $(M, \psi)$ to $(X, d)$ is less than $(2 h+7 \varepsilon)$.

First, let $x \in M$ be outside of the tubular neighborhood and $s$ be a geodesic of length less than $\varepsilon$ in $(M, \phi)$ connecting $x$ and $x_{i}$. Let $x_{i}^{\prime}$ be a point outside the tubular neighborhood which is within $\varepsilon$ of $x$ in the original $\phi$-metric. This is a condition on how thin the tubes should be. The triangle inequality then shows that $\psi\left(x_{i}, x\right)<h+3 \varepsilon$. completing the case in which $x$ is outside of the tubular neighborhood. If $x$ is inside of the tubular neighborhood, we can get outside by moving a distance $(h+2 \varepsilon)$ after which we can find a vertex within $(h+3 \varepsilon)$ units, as before. This shows that the vertex set is $(h+5 \varepsilon)$ dense in $(M, \psi)$. Notice that we have proven slightly more than we have said. If $x \in(M, \psi)$, then we can find a vertex $x_{i}$ so that $\psi\left(x, x_{i}\right)<2 h+5 \varepsilon$, $\phi\left(x, x_{i}\right)<\varepsilon+r<2 \varepsilon$ and so that $d\left(f\left(x_{i}\right), f(x)\right)<\varepsilon$.

We use this to prove the inequality 
since for vertices $x_{i}$ and $x_{j}$ close to $x$ and $y$ as above we have

$$
\begin{aligned}
\psi(x, y) & <\psi\left(x, x_{i}\right)+\psi\left(x_{i}, x_{j}\right)+\psi\left(x_{j}, y\right) \\
& <(2 h+5 \varepsilon)+d\left(f\left(x_{i}\right), f\left(x_{j}\right)\right)+2 h+5 \varepsilon \\
& <d\left(f\left(x_{i}\right), f(x)\right)+d(f(x), f(y))+d\left(f(y), f\left(x_{j}\right)\right)+4 h+10 \varepsilon \\
& <\varepsilon+d(f(x), f(y))+\varepsilon+4 h+10 \varepsilon=d(f(x), f(y))+4 h+12 \varepsilon .
\end{aligned}
$$

Choosing $\varepsilon$ small enough and combining $(* *)$ and $(* * *)$ and smoothing the metric, we see that for any $i$ there exists a Riemannian metric $\psi_{i}$ on $M$ such that

$$
\left|\psi_{i}(x, y)-d(f(x), f(y))\right|<\frac{1}{i}
$$

This completes the proof of (i).

Let $\rho$ be a contractibility function for $(X, d)$. Using basic lifting properties [Mo] of $f$ we see that the metric space $\left(M, \psi_{i}\right)$ has a contractibility function $\rho_{i}$ satisfying

$$
\rho_{i}(t)<\rho\left(t+\frac{1}{i}\right)+\frac{1}{i} .
$$

On the other hand for $t$ less than the radius of injectivity $R_{i}$ of the Riemannian manifold $\left(M, \psi_{i}\right)$ we have

$$
\rho_{i}(t)=t \text {. }
$$

Combining these two properties it is easy to see that the function $\rho^{\prime}$ defined by

$$
\rho^{\prime}(t)=\rho\left(t+\frac{1}{i}\right)+\frac{1}{i}+R_{i} \text { if } t \in\left[R_{i+1}, R_{i}\right)
$$

is a common contractibility function for all $\left(M, \psi_{i}\right)$ (we assume here that $\left\{R_{i}\right\}$ monotonely decreases to 0 ).

In words, what we did was to rescale the metric on $M$ to make it bigger than the metric on $X$ and then alter the metric on $M$ in a neighborhood of a 1-dimensional set to make the metric there look like the metric on a 1-skeleton approximating $X$. We also inserted a barrier of height $h$ between this new region and the old external region.

The result is that the 1 -dimensional set is $(h+3 \varepsilon)$-dense in the new metric on $M$. The shortest paths between points in the 1 -dimensional set lie in the one-dimensional set because $h$ is large enough (and the metric on $M$ is big enough) that there is no advantage to be gained by crossing the barrier to go outside of the 1-dimensional neighborhood.

Corollary 5. A path metric on a closed smoothable topological manifold can be approximated arbitrarily closely by a Riemannian metric.

Proof. This is the case $f=\mathrm{id}$.

Remark 6. (i) As a special case of Corollary 5, we see that if $(M, \psi)$ is a Riemannian manifold and $\Sigma$ is any smooth structure on $M$, then there is a sequence of Riemannian metrics $\left(M, \phi_{i}\right)$ compatible with $\Sigma$ so that $\lim \left(M, \phi_{i}\right)=(M, \psi)$ in $\mathscr{M}^{\operatorname{man}}(n, \rho)$ for some $\rho$. This emphasizes that convergence on Gromov-Hausdorff space is a $C^{0}$ phenomenon. In special cases, we can see this directly. If $\Sigma_{1}$ is a homotopy sphere and $\Sigma_{2}$ is any other homotopy sphere, then $\Sigma_{2}$ is diffeomorphic to $\Sigma_{1} \# \Sigma_{3}$ for some $\Sigma_{3}$. Therefore, a 
sequence of $\Sigma_{1} \# \Sigma_{3}$ 's in which the $\Sigma_{3}$ 's get smaller and smaller gives a sequence of Riemannian manifolds diffeomorphic to $\Sigma_{2}$ converging to $\Sigma_{1}$. One might compare this with $\S 2$ of Essay II of [KS] where it is shown that if $M$ and $N$ are homeomorphic smooth manifolds, then diffeomorphisms $M \rightarrow N$ are $C^{0}$ dense in any component of $\operatorname{Homeo}(M, N)$ which contains a diffeomorphism.

(ii) It is interesting to compare case (i) of our theorem with the results of [C], where it is shown that every path metric can be approximated by Riemannian metrics on different 2-manifolds. In contrast, we show that if $X$ is the $U V^{0}$ image of $M^{n}, n \geq 3$, then $X$ can be approximated by a sequence of different metrics on Riemannian manifolds diffeomorphic to $M$. There is an extensive literature on $U V^{0}$ (or monotone) mappings of manifolds. See [Be, F, W].

Corollary 7. There exist a compactum $X$, a contractibility function $\rho$, and nonhomeomorphic manifolds $M$ and $N$ so that for every $\varepsilon>0$ there are Riemannian metrics $\phi_{\varepsilon}$ and $\psi_{\varepsilon}$ on $M$ and $N$, respectively, so that $\left(M, \phi_{\varepsilon}\right)$ and $\left(N, \psi_{\varepsilon}\right)$ are $\varepsilon$-close to $X$ in $\mathscr{C} \mathscr{M}$.

Proof. The main theorem of [DF] shows that there are nonhomeomorphic manifolds $M$ and $N$ and cell-like maps $M \stackrel{\mathrm{CE}}{\rightarrow} X$ and $N \stackrel{\mathrm{CE}}{\rightarrow} X$. The rest follows from the main result of this paper.

Remark 8. The manifold $M$ above need not be very complicated. In particular, an $M$ with the desired properties can be obtained by attaching $D^{4}$ to $S^{3}$ by a degree 3 map, embedding the resulting polyhedron on $\mathbb{R}^{7}$, and taking the double of a regular neighborhood. The manifold $N$ is a manifold homotopy equivalent to $M$ which is produced by varying 3-torsion in the $K$-theoretic characteristic classes of the tangent bundle.

\section{THE NON-COMPACT CASE}

The argument given above clearly does not work when the diameter $D$ of $X$ is infinite. In $\S 2$ of [C], the Cassorla shows that for $X$ compact and $n$ large, the 1-complex obtained by taking a $10^{-n}$-dense set $\left\{x_{i}\right\}$ in $X$ and connecting vertices whose $X$-distance is less than $5^{-n}$ gives a good metric approximation to $X$.

In the noncompact case, repeating his construction while letting $n$ be a function of $x$ which grows as $x \rightarrow \infty$ in $X$ gives a good uniform approximation to $X$ by a 1-complex whose edges have bounded lengths. Repeating our construction using this 1-complex gives a Riemannian manifold uniformly approximating the original path space $X$.

The main application we have in mind for this is to the case in which $X$ is a smoothable manifold with a topological path metric which is uniformly contractible in the sense of [G, DFW]. The construction of this paper is then used to find a uniformly contractible Riemannian metric on $X$ which is coarsely equivalent to the path metric.

\section{BIBLIOGRAPHY}

[Be] M. Bestvina, Characterizing $k$-dimensional universal Menger compacta, Mem. Amer. Math. Soc., vol. 71, Amer. Math. Soc., Providence, RI, 1988.

[B] R. H. Bing, Partitioning continuous curves, Bull. Amer. Math. Soc. 58 (1952), 536-556. 
[C] M. Cassorla, Approximating compact inner metric spaces by surfaces, Indiana Univ. Math. J. 41 (1992), 505-513.

[D] A. N. Dranishnikov, On resolutions of $L C^{n}$-compacta, Shape Theory and Geometric Topology (S. Mardesic and J. Segal, eds.), Lecture Notes in Math., vol. 1283, Springer-Verlag, Berlin and New York, 1987, pp. 48-59.

[DF] A. N. Dranishnikov and S. C. Ferry, Cell-like images of topological manifolds and limits of manifolds in Gromov-Hausdorff space, preprint.

[DFW] A. N. Dranishnikov, S. Ferry, and S. Weinberger, Large Riemannian manifolds which are flexible, preprint.

[DW] J. Dydak and J. J. Walsh, Infinite dimensional compacta having cohomological dimension two: An application of the Sullivan Conjecture, Topology 32 (1993), 93-104.

[F] S. Ferry, Constructing UV $U V^{k}$-maps between spheres, Proc. Amer. Math. Soc. 120 (1994), 329-332.

[G] M. Gromov, Large Riemannian manifolds, Lecture Notes in Math., vol. 1201, SpringerVerlag, Berlin and New York, 1986, pp. 108-122.

[KS] R. Kirby and L. C. Siebenmann, Foundational essays on topological manifolds, smoothings, and triangulations, Princeton Univ. Press, Princeton, NJ, 1977.

[L] R. C. Lacher, Cell-like mappings and their generalizations, Bull. Amer. Math. Soc. 83 (1977), 495-552.

[Mo] T. Moore, Gromov-Hausdorff convergence to non-manifolds, J. Geometric Anal. (to appear).

[P] P. Peterson, V, A finiteness theorem for metric spaces, J. Differential Geom. 31 (1990), 387-395.

[S] S. Smale, A Vietoris mapping theorem for homotopy, Proc. Amer. Math. Soc. 8 (1957), 604-610.

[W] J. Walsh, Isotoping mappings to open mappings, Trans. Amer. Math. Soc. 250 (1979), 121-145.

Department of Mathematical Sciences, State University of New York at BinghamTON, Binghamton, New YORK 13901 